\title{
Loads due to Stray Microwave Radiation in ITER
}

\author{
Johan W. Oosterbeek ${ }^{\mathrm{a}, \mathrm{b}}$, Victor S. Udintsev ${ }^{\mathrm{a}}$, Franco Gandini ${ }^{\mathrm{a}}$, Matthias Hirsch ${ }^{\mathrm{c}}$, Heinrich P. Laqua ${ }^{\mathrm{c}}$, \\ Nick Maassen ${ }^{\mathrm{b}}$, Yunxing Ma ${ }^{\mathrm{a}}$, Alexei Polevoi ${ }^{\mathrm{a}}$, Antoine Sirinelli ${ }^{\mathrm{a}}$, George Vayakis ${ }^{\mathrm{a}}$, Mike J. Walsh ${ }^{\mathrm{a}}$ \\ ${ }^{a}$ ITER Organization, Route de Vinon-sur-Verdon, CS 90 046, 13067 St. Paul Lez Durance Cedex, France. \\ ${ }^{b}$ Eindhoven University of Technology, P.O. Box 513, 5600 AZ, Eindhoven, The Netherlands. \\ ${ }^{c}$ Max-Planck-Institut für Plasmaphysik, EURATOM Association, Teilinstitut Greifswald, D-17489 Greifswald, Germany
}

\begin{abstract}
High-power microwaves generated by gyrotrons will be extensively used in ITER for a variety of purposes such as assisting plasma breakdown, plasma heating, current drive, tearing mode suppression and as a probing beam for the Collective Thomson Scattering diagnostic. In a number of these schemes absorption of the microwaves by the plasma will not be full and in some cases there could be no absorption at all. This may result in a directed beam with a high microwave power flux or - depending on location and plasma conditions - an approximately isotropic microwave power field. The contribution of electron cyclotron emission to these power densities is briefly discussed. Exposure to in-vessel components leads to absorption by metals and ceramics. In this paper microwave power densities are estimated and, following a brief review of absorption, thermal loads on in-vessel components are assessed. The paper is concluded by a discussion of the current approach to control such loads.
\end{abstract}

Keywords: microwave stray radiation, gyrotron, power density, absorption, ECRH, ECE loss

\section{Introduction}

Microwave power densities in ITER may vary from hundreds of $\mathrm{MW} / \mathrm{m}^{2}$ to several $\mathrm{kW} / \mathrm{m}^{2}$ depending on whether the power originates from directed gyrotron beams, stray radiation by diffused non-absorbed gyrotron power or by Electron Cyclotron Emission (ECE) escaping from the plasma. In this paper the focus is on loads originating from gyrotrons but a brief discussion on loads by ECE is included in order to place this into context.

At ITER $20 \mathrm{MW}$ of microwave power @ $170 \mathrm{GHz}$ will be installed for Electron Cyclotron Resonance Heating (ECRH), Electron Cyclotron Current Drive (ECCD) and start-up assist [1]. These sources will be referred to as ECW. In addition a 1 MW gyrotron @ 60 $\mathrm{GHz}$ is foreseen as probing beam for Collective Thomson Scattering (CTS). The probing beam will be modulated on-off by $50 \%$. The CTS power is modest compared to the power of the ECW system but the load is still large as the frequency is selected such that the microwave beam is scattered opposed to being absorbed.

Microwave power by ECE increases strongly with increase in electron temperature. To find an estimate of the ECE loading, data from a study on a high performance ITER discharge is considered.

This paper is structured as follows. Power densities by directed beams are assessed. The parameters of Gaussian beams are briefly reviewed and illustrated with the data of the CTS system. This is followed by a description of ECW directed beams. Stray radiation is assessed by means of simplified model of the vessel interior. A brief discussion on ECE losses is given. Absorption is discussed in order to assess the loads.

\section{Assessment of power densities}

\subsection{Directed beam, CTS}

The size of the beam is measured by beam radius $w$ which is the radius where the E-field has fallen to $1 / \mathrm{e}$ with respect to the value on axis and is given by [2].

$$
w=w_{0}\left[1+\left(\frac{\lambda z}{\pi w_{0}^{2}}\right)^{2}\right]^{0.5},
$$

with $\mathrm{w}_{0}$ the waist where $w$ is minimal, $\lambda$ the wavelength and $\mathrm{z}$ the propagation distance. The beam in general is astigmatic giving rise to two orthogonal beam radii $\mathrm{w}_{\mathrm{x}}$ and $\mathrm{w}_{\mathrm{y}}$. Fig. 1 shows a cartoon in the poloidal plane of the conceptual CTS system and Fig. 2 the evolution of the beam radii. The beam is launched @ $\mathrm{z}=0$ with a waist of $20.3 \mathrm{~mm}$, propagates over a series of curved mirrors in the port plug and propagates through the vessel where it bounces off the High Field Side (HFS) blanket and back to the Low Field Side (LFS).

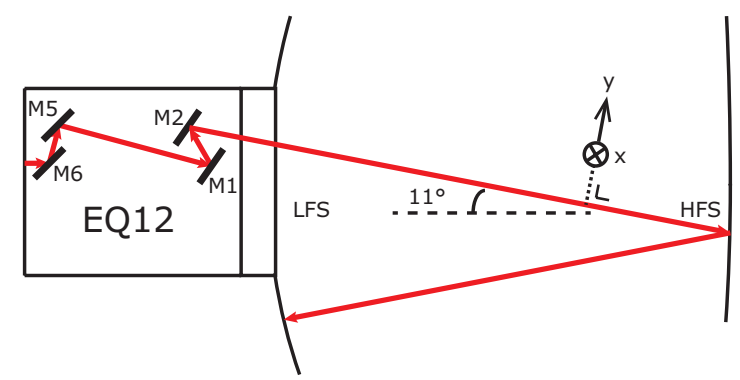

Fig 1. Poloidal view of the center of the CTS probing beam.

The profile of the actual power density for a given beam radius $w$ and total power $\mathrm{P}_{\text {tot }}$, with $\mathrm{r}$ being the radial distance along the profile, is given by equation 2 [2].

$$
P_{a c t}=P_{t o t} \frac{2}{\pi w^{2}} \exp \left[-2\left(\frac{r}{w}\right)^{2}\right]
$$


In case of an astigmatic beam the substitution $\mathrm{w}^{2}=\mathrm{w}_{\mathrm{x}}{ }^{*} \mathrm{w}_{\mathrm{y}}$ is made. Fig. 3 shows the profile on the HFS $\left(@ \mathrm{z}=9.9 \mathrm{~m}, \mathrm{P}_{\text {tot }}\right.$ in plasma $=0.8 \mathrm{MW}$ ) resulting in a peak of $25 \mathrm{MW} / \mathrm{m}^{2}$. A similar analysis on return at the LFS (@ $\mathrm{z}=14.4 \mathrm{~m}$ ) gives $3 \mathbf{M W} / \mathbf{m}^{2}$. The numbers are a slight over-estimate as a toroidal injection angle of $7^{\circ}$ is used and a poloidal injection angle of $11^{\circ}$.

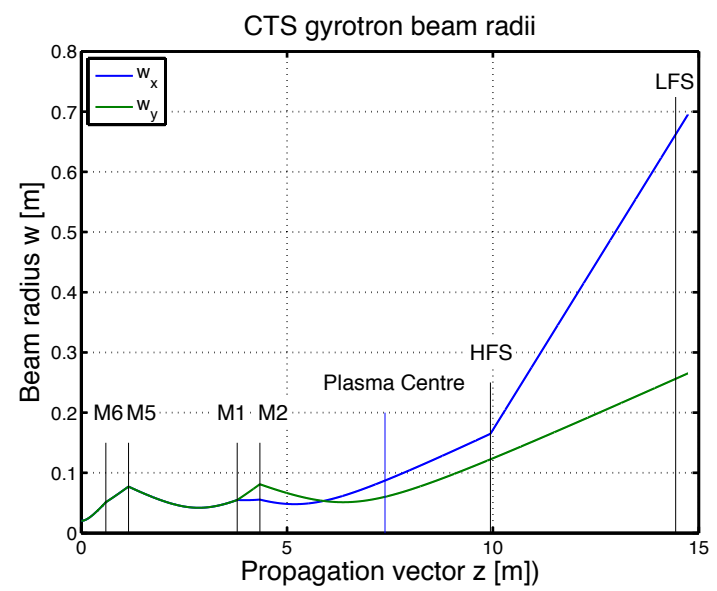

Fig 2. Evolution of the beam radii of the CTS probing beam. The propagation vector start at the closure plate with a waist $\mathrm{w}_{0}=20.3 \mathrm{~mm}$. The waist is transformed following the beam trajectory z. The component locations and vessel references are mapped onto the propagation vector. On reflection off the HFS the horizontal waist diverges additionally due to the toroidal vessel curvature $(\mathrm{R} \sim 6-2=4 \mathrm{~m})$.

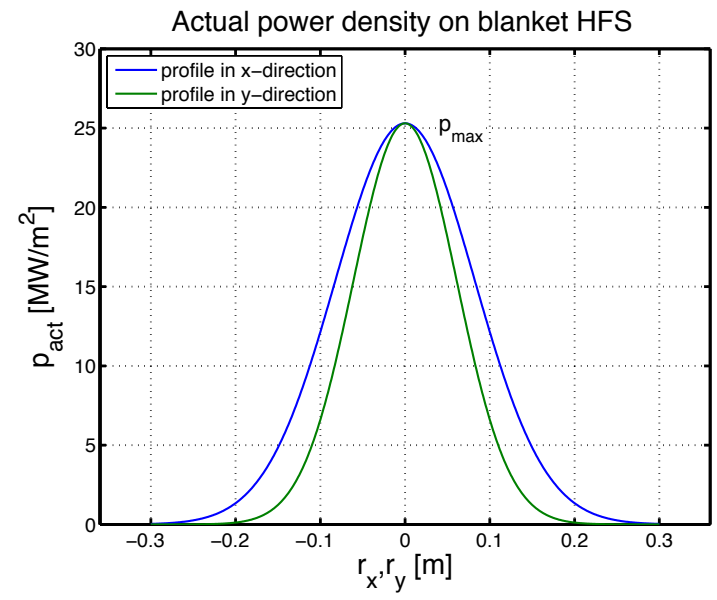

Fig 3. Power density profile of the CTS beam at the HFS. The peak power density is high but falls off quickly.

\subsection{Directed beam, ECW}

\subsubsection{Start-up assist}

An assessment of directed beam for start-up assist is given by Gandini [3]. At the time of writing however, design constraints have altered the scenario from equatorial launch to upper launch. During the first ITER phase, with no blanket, power from 8 gyrotrons @ $840 \mathrm{~kW}$ is coupled to two steerable mirrors in the upper launcher in port 16. These two mirrors launch two synthesized beams with a total beam power of 6.7 MW in vessel with an equivalent waist of $\sim 4 \mathrm{~cm}$ at the $\mathrm{HFS}$. At the HFS a large reflector, order $0.5 \times 1 \mathrm{~m}$, will be mounted to reflect the non-absorbed power into equatorial port plug 17 . This port will initially not be used and will contain a custom built dump that must cope with non-absorbed power until plasma break-down is achieved. Later, during operation with the blanket and with all 4 upper launcher available, a scheme is envisaged to reflect off the blanket tiles, order $1 \mathrm{~m}$, but distributing the power over four launchers while depositing on the same flux surfaces. In either scheme stray radiation is at maximum $3 \mathrm{MW} / \mathrm{m}^{2}$ during $5.5 \mathrm{~s}$.

\subsubsection{Polarization mismatch.}

At nominal plasma operation ECW beams are launched from both equatorial and upper ports depending on physics requirements. Injection is aligned to first harmonic O-mode (O1) [4] at deposition for optimum absorption. Any fraction accidentally coupled to $\mathrm{X} 1$ reflects at the $\mathrm{X} 1$ cutoff layer at the low field side and thus back at the LFS. In practice one can never avoid a slight mismatch in polarization. Latest estimates are that a mismatch can be kept below $3^{\circ}$. This would lead to a non-absorbed fraction in the order of $1 \%$. This power reflects and refracts away as a directed beam. To assess this consider the following scenario: $1 \mathrm{MW}$ beam, waist $3 \mathrm{~cm} @$ at $\mathrm{z}=2.5 \mathrm{~m}, 1 \%$ of power in $\mathrm{X} 1$ and the $\mathrm{X} 1$ cutoff at $1 \mathrm{~m}$ from the launcher. Considering a straight trajectory the $1 \%$ of power travels just $2 \mathrm{~m}$ before it is back at the LFS, i.e. approximately the same distance as from launcher to the waist. In the waist the power density is $0.6 \mathrm{GW} / \mathrm{m}^{2}$, the $1 \%$ refracted beam at the LFS is $\sim 6 \mathrm{MW} / \mathrm{m}^{2}$.

\subsection{Stray radiation power density, ECW}

After many reflections of the directed beam, and scrambling by the plasma, a stray radiation field builds up. The power distributes over the entire vessel and interacts with all components. A power balance can be made up $[5,8]$ which is given in equation 3 .

$$
p=P_{\text {tot }} \frac{1-\alpha_{p}}{\alpha_{w} A_{w}+\left\langle\alpha_{p}\right\rangle A_{p}}
$$

In the expression $p$ is the average power density, $\alpha_{\mathrm{p}}$ is the single pass plasma absorption, $\alpha_{\mathrm{w}}$ the absorbed fraction of power of the wall $\left(\alpha_{w}=1-R_{w}\right.$, with $R_{w}$ the reflected fraction of power) and $A_{w}$ the surface area of the wall. The wall surface is set to $900 \mathrm{~m}^{2}$ from which $43 \mathrm{~m}^{2}$ are apertures. The product $\alpha_{\mathrm{w}} \mathrm{A}_{\mathrm{w}}$ is in fact the sum of many loss areas $\alpha_{n} A_{n}$, inclusive apertures for which $\alpha_{n}$ is set to unity. $\left\langle\alpha_{p}\right\rangle$ is the average isotropic plasma reabsorption, set to 0.1 and $A_{p}$ the plasma surface set to $680 \mathrm{~m}^{2}$. The input power is set to $20 \mathrm{MW}$ and first pass plasma absorption is set to $95 \%$, i.e. $1 \mathrm{MW}$ of nonabsorbed ECW after the first pass. Taking absorbed fractions for the wall as calculated in section 3 , one finds $\mathrm{p}$ of the order of $10 \mathrm{~kW} / \mathrm{m}^{2}$. Without absorption by the plasma one finds $\mathrm{p}=0.4 \mathrm{MW} / \mathrm{m}^{2}$. Application to CTS, at $60 \mathrm{GHz}$, with $0.5 \mathrm{MW}$ average power and no plasma absorption, yields $\mathrm{p}=7 \mathrm{~kW} / \mathrm{m}^{2}$. 
It is noted that a very high wall refection $R_{w}$ of 0.95 is found. In practive $R_{w}$ will be lower and a detailed analysis of the vessel interior is required.

While good results are obtained with the power balance at the MISTRAL test facility in Greifswald [6, 7], care should be taken when applied to ITER. The interior of the MISTRAL is simple and has been optimized for isotropic stray radiation. In contrast, the torus contains cavities, such as deep ports and the space between blanket and vessel, leading to local variations in power $[8,9]$.

\subsection{Electron cyclotron wave power loss.}

ECE losses scale with $\mathrm{n}_{\mathrm{e}}^{0.5 *} \mathrm{~T}_{\mathrm{e}}{ }^{2.5}$, with $\mathrm{n}_{\mathrm{e}}$ the electron density and $\mathrm{T}_{\mathrm{e}}$ the electron temperature [10]. To obtain a coarse estimate on the losses, data from a study of an ITER 15 MA reference discharge [11] was used. In this study equilibrium data was fed into a Trubnikov expression [10],

$$
\mathrm{P}_{\text {syn }}=1.310^{-7} \mathrm{n}_{\mathrm{e}} \mathrm{T}_{\mathrm{e}}<\mathrm{T}_{\mathrm{e}}>{ }^{1.5} \mathrm{~B}^{2} \Phi,
$$

with $\Phi=\left(\mathrm{B}\left(1+18 \mathrm{aR}_{\mathrm{L}}^{-1}<\mathrm{T}_{\mathrm{e}}>^{-0.5}\right)\left(1-\mathrm{R}_{\mathrm{w}}\right)<\mathrm{n}_{\mathrm{e}}>^{-1} \mathrm{a}^{-1}\right)^{0.5}$, $\mathrm{P}\left[\mathrm{MW} / \mathrm{m}^{3}\right], \mathrm{T}_{\mathrm{e}}[\mathrm{keV}], \mathrm{n}_{\mathrm{e}}\left[10^{19} \mathrm{~m}^{-3}\right]$, quantities between $<>$ volume average values, toroidal magnetic field, $\mathrm{B}=5.3 \mathrm{~T}$, minor and major radii, $\mathrm{a}=2 \mathrm{~m}, \mathrm{R}_{\mathrm{L}}=6.2 \mathrm{~m}$, and a wall reflection $\mathrm{R}_{\mathrm{w}}=0.6$ [11]. Applying the units above, taking $\mathrm{T}_{\mathrm{e}}=25,\left\langle\mathrm{~T}_{\mathrm{e}}\right\rangle=10, \mathrm{n}_{\mathrm{e}}=10$ and integrating over the plasma volume emission an ECE loss of $\sim 13 \mathrm{MW}$ was obtained. Using the same methodology as for the ECW stray calculation, with the loss areas increased to generate $\mathrm{R}_{\mathrm{w}} \sim 0.6$ this leads to power densities of $36 \mathrm{~kW} / \mathrm{m}^{2}$. For the same conditions as for the ECW calculation ( $\mathrm{Rw} 0.95)$, the ECE loss decreases to $5 \mathrm{MW}$ but the power density increases to $110 \mathrm{~kW} / \mathrm{m}^{2}$ as would be expected in the non-lossy regions.

It is concluded that frequency integrated ECE loss is well in excess of isotropic ECW stray radiation, ranging in the tens of $\mathrm{kW} / \mathrm{m}^{2}$. However, as all parameters are stringly frequency dependent, a more detailed study is needed for a precise answer.

\section{Absorption}

Absorption is by lossy metals and ceramics. Effects by polarization, in the case of oblique incidence (Fresnel equations) are not discussed but are included in the margins set in this section.

For metals, assuming the transmitted fraction is negligible, the absorbed fraction of power at normal incidence is $4 / Z_{0} \sqrt{\pi f \mu_{0} \rho}$ [2] with $Z_{0}$ the impedance of free space, $f$ the frequency, $\mu_{0}$ the permeability of free space, and $\rho$ the resistivity. In practice the absorbed fraction is increased by surface roughness [12] and a factor 1.5 has been applied. It is noted that plasma coating may increase this to a factor 3 [12]. Also: the manufacturing finish of each metal should be applied. Absorbed fractions for some typical materials at $300^{\circ} \mathbf{C}$ and at $170 \mathrm{GHz}$ are: Beryllium: $0.4 \%$, Tungsten, $0.5 \%$, Stainless Steel (Vacuum Vessel, 316L(N)-IG) $1.3 \%$.

For ceramics the single pass absorbed fraction of power $\quad \tau=\left(1-\Gamma^{2}\right)(1-\exp (-2 \alpha \mathrm{d}))$ with $\Gamma=$ $\left(1-\sqrt{\varepsilon_{\mathrm{r}}^{\prime}}\right) /\left(1+\sqrt{\varepsilon_{\mathrm{r}}^{\prime}}\right)$ with $\varepsilon_{\mathrm{r}}^{\prime}$ the relative permittivity, $\mathrm{d}$ the thickness and $\alpha$ the field attenuation constant as defined in the propagation constant $\gamma=\alpha+j \beta$ (with phase constant $\beta$ ). For low loss ceramics $\alpha$ is approximated by $\frac{\pi}{\lambda_{0}} \sqrt{\varepsilon_{\mathrm{r}}^{\prime}} \cdot \tan \delta$ with $\lambda_{0}$ the vacuum wavelength and tan $\delta$ the loss tangent $[13,14]$. It is not always trivial to obtain tan $\delta$ at high frequencies and one must sometimes revert to dedicated measurements [15]. Multiple internal reflections increase the single pass absorption. Also, in case the sample is surrounded by metal walls, as in a microwave oven, the absorbed fraction becomes $\tau /[1-R(1-\tau)]]$ with $R$ the wall reflectivity. As an example a vacuum quartz window by Heraeus, Infrasil30 $1^{\mathrm{TM}}: \varepsilon_{\mathrm{r}}^{\prime} \sim 3.8$ and $\tan \delta \sim 2.9 \mathrm{E}-4$ [16]. For d = 9.95 mm (made 'resonant' @ $170 \mathrm{GHz}: \Gamma=0$ ) $\tau=2 \%$. But internal reflections increase the fraction: for a window with single incident beam this is approximated by $\mathrm{A} \approx \pi \mathrm{fdtan} \delta\left(1+\varepsilon_{\mathrm{r}}^{\prime}\right) / \mathrm{c}=2.5 \%[16,17]$. Ceramics as approved in the ITER vacuum handbook should still be used with consideration as loss tangents are of the order of $1 \mathrm{E}-3$. Depending of material, frequency and location the major fraction of incident power may be absorbed.

\section{Evaluation of loads}

Using the absorption coefficients in section 3 a microwave load of $1 \mathrm{MW} / \mathrm{m}^{2} @ 170 \mathrm{GHz}$ is estimated to result in a $4 \mathrm{~kW} / \mathrm{m}^{2}$ heat load on the Be-wall, $13 \mathrm{~kW} / \mathrm{m}^{2}$ on the Stainless Steel vessel wall and $5 \mathrm{~kW} / \mathrm{m}^{2}$ heat load on the Tungsten divertor. At $60 \mathrm{GHz}$ these numbers are to be multiplied by $(60 / 170)^{0.5}$.

Ceramics require individual assessment, but as an example: a $1 \mathrm{MW} / \mathrm{m}^{2}$ flux @ $170 \mathrm{GHz}$ incident on a quartz disk described in section 3 with a diameter of $63 \mathrm{~mm}$ and a thickness of $10 \mathrm{~mm}$, deposits $77 \mathrm{~W}$ into the disk. The increase in temperature is a volume effect and only depends on material properties and frequency. Considering no cooling the rise in temperature is $1.2 \mathrm{~K} / \mathrm{s}$.

\subsection{Loads by directed beam}

During start-up assist a maximum power density of $3 \mathrm{MW} / \mathrm{m}^{2} \mathrm{LFS}$ has been ensured resulting in a peak heat load of $12 \mathrm{~kW} / \mathrm{m}^{2}$ on the Be-wall. This load will be present during $5.5 \mathrm{~s}$ at maximum and is likely to reduce as the plasma forms.

During plasma operation a mismatch in polarization could result in a randomly located beam with a peak power of $6 \mathrm{MW} / \mathrm{m}^{2}$ per MW beam power on the LFS in case of $1 \%$ launch in $\mathrm{X} 1$. On the Be-wall this presents a heat load of $24 \mathrm{~kW} / \mathrm{m}^{2}$. This can be handled and will drop off quickly after a few passes. However, the initial pass may also be incident on a gap between tiles giving 
rise to risk of arcing, or be incident on a port plug where the large $\mathrm{CW}$ power may lead to excessive temperatures of windows and thin metal coatings. Referring to the calculation example in 2.2.2, an accidental mistake in polarization setting altogether could direct a microwave beam with a peak power $0.6 \mathrm{GW} / \mathrm{m}^{2}$ and a beam radius of several $\mathrm{cm}$ on the LFS or into a port plug. It is advised to study beam refraction and the regions at risk and to install diagnostics in the vicinity of these locations that can reliably and quickly detect refracted beams.

For CTS ( $50 \%$ duty, $60 \mathrm{GHz}$ ) the peak heat loading on the HFS is estimated to be $30 \mathrm{~kW} / \mathrm{m}^{2}, 4 \mathrm{~kW} / \mathrm{m}^{2} \mathrm{LFS}$.

\subsection{Loads by stray radiation}

The thermal wall loading by stray radiation as found in section 2.3 is of the order of $50 \mathrm{~W} / \mathrm{m}^{2}$ - while already using a high wall reflectivity - and can be neglected.

As briefly discussed in 2.4, EC-losses are in this respect much more relevant. At $\mathrm{Te}=25 \mathrm{keV}$ the EClosses are already a multiple of the stray radiation. While the wall load due to ECE-losses will be much lower as compared to directed beam it must be investigated further as it impinges on all components $\mathrm{CW}$.

\section{Conclusion and present situation}

At start-up very high power densities are expected but these are well localized and last up to $5.5 \mathrm{~s}$. Measures are in place to reduce the microwave power density to $3 \mathrm{MW} / \mathrm{m}^{2}$ on the LFS.

It is concluded that the risk of a high power refracted beam by ECW, due to a mismatch in polarization or wrong polarization setting, is a substantial risk due to the extreme power density of the focused beam. This risk must be mitigated by further study and diagnostics capable of detecting such events.

The thermal loads due to stray radiation levels by ECW and CTS are not excessive and can be handled by the first wall. But one should be vigilant in areas behind the blankets and in port plugs where less heat sink is generally present and where ceramics may be used.

It is shown that in high performance discharges the ECE loss from the plasma dominates the nominal stray radiation from ECW and CTS.

To monitor microwave power levels, microwave detectors are presently designed [18]. They are tuned to handle up to $3 \mathrm{MW} / \mathrm{m}^{2}$ for $5.5 \mathrm{~s}$, can take $1.25 \mathrm{MW} / \mathrm{m}^{2}$ $\mathrm{CW}$, while also capable of detecting several $\mathrm{kW} / \mathrm{m}^{2}$ power densities with an integration time of $60 \mathrm{~s}$.

A collaboration between Eindhoven University of Technology and W7-X exists to model and measure stray radiation as well as absorption by stray radiation and directed beam. The MISTRAL vessel is utilized and a medium size resonator is being prepared to measure absorption coefficients of ITER in-vessel materials.

\section{Disclaimer}

The views and opinions expressed herein do not necessarily reflect those of the ITER Organization.

\section{References}

[1] C. Darbos et al., ECRH system for ITER, AIP Conf. Proc. 1187, 531 (2009).

[2] Paul F. Goldsmith, Quasioptical Systems, IEEE Press, New York (1998).

[3] F. Gandini et al., Stray Power Estimates from EC Exploitation during ITER Plasma Operations, AIP Conf. Proc 1406, 173 (2011).

[4] G. Ramponi et. al., Physics analysis of the ITER ECW system for optimized performance, Nucl. Fusion 48 (2008) 054012.

[5] H.J. Hartfuss, Problems with ECRH stray radiation in Wendelstein 7-X, 30-th EPS, Vol. 27A, O-3.2C

[6] M. Hirsch et al., The Impact of Microwave Stray Radiation to In-Vessel Diagnostic Components, International Conf. on Fusion Reactor Diagnostics, Varenna, 9 - 13 September 2013

[7] D. Hathiramani, Microwave stray radiation: Measures for steady state diagnostics at Wendelstein W7-X, Fusion Engineering and Design 88 (2013) 1232-1235.

[8] H. Laqua et al., Distribution of the ECRH stray radiation in fusion devices, EPS Conference Proceedings, Funchal, Madeira, ECA Vol. 25A, 1277 (2001) .

[9] M. Hirsch, Major results from the Stellarator Wendelstein 7-AS, Plasma Phys. Control. Fusion 50 (2008) 053001.

[10] B.A. Trubnikov, in Review of Plasma Physics,Vol.7 (Leontovich, M.A. Ed.), Consultants Bureau, New York (1979) 345

[11] A.R. Polevoi, et al., J. Plasma Fusion Res. 5, 82-87 (2002) , http://www.jspf.or.jp/JPFRS/index_vol5.html

[12] W. Kasparek, Three-mirror resonator reflectivity measurement of plane and grooved surfaces: Setup, options, results, 6th European Conference on Antennas and Propagation (EUCAP), 2012, p 585 - 589.

[13] C.A. Balanis, Advanced Engineering Electromagnetics, John Willey \& Sons (1989).

[14] S. Ramo et al., Fields and Waves in Communication Electronics, John Wiley \& Sons (1994).

[15] R. Heidinger et al., Dielectric property measurements at elevated temperatures based on open resonators, Proceedings MIOP, Stuttgart, 2001, pp 186-90.

[16] J.W. Oosterbeek et al., A Line-of-Sight Electron Cyclotron receiver for electron cyclotron heating feedback control of tearing modes, Rev. of Scientific Instr, 78, 093503 (2008).

[17] H.-U. Nickel, Hochfrequenztechnische Aspekte zur Entwicklung rückwirkungsarmer Ausgangsfenster für Millimeterwellengyrotrons hoher Leistung, FZ Karlsruhe, FZKA Report No. 5513 (1995).

[18] N.A. Maassen, Microwave detector design for ITER, Internship Master program Applied Physics, Eindhoven University of Technology, The Netherlands, 2014. 